\title{
Una Fe qUe haga JUSTICIA: Orientaciones Religiosas Y SUS CORRELATOS SOCIALES
}

\section{A FAITH WHICH DOES JUSTICE: RELIGIOUS ORIENTATIONS AND THEIR SOCIAL CORRELATES}

\author{
Dr. Frank Bernt \\ Saint Joseph's University (USA)
}

\begin{abstract}
Resumen: Se presenta un panorama de las investigaciones que vinculan las medidas de orientación religiosa con las actitudes y conductas sociales y antisociales. Se consideran además, por un lado, los resultados de varias escalas de religiosidad y por el otro, su relación con las que registran actitudes y conductas prosociales y antisociales (i.e., caridad y altruismo versus prejuicio y discriminación). Como conclusión, se incluyen algunas implicaciones, así como, se proponen algunas direcciones para investigaciones futuras sobre dicha relación.
\end{abstract}

Palabras Claves: Orientación Religiosa, Altruismo, Prejuicio

\begin{abstract}
The paper presents an overview of studies that link various measures of religious orientation with prosocial and anti-social attitudes and behaviors, including, on one hand, various scales of religiosity, and on the other, prosocial and antisocial attitudes and behaviors (for example, charity and altruism, prejudice and discrimination). The conclusion includes several implications as well as directions for future studies in this area.
\end{abstract}

Key Words: Religious Orientation, Altruism, Prejudice

La realidad compleja de la religión -tanto en el mundo histórico como en el mundo actual tiene dos caras, o sea, es una realidad ambivalente. Por un lado, se valora como fuente de caridad y justicia; por otro, se repudia como causa de guerras, exclusión y toda manera de injusticia. Entre los variados debates al respecto, quienes están a cargo de las ciencias sociales suponen que, dicha complejidad se basa no necesariamente en la naturaleza de la realidad divina, sino en la respuesta humana. Quizás hay sólo una Fe verdadera; sin embargo, hay una diversidad de Fes individuales o sea, de respuestas humanas a la experiencia religiosa quizás tantas variedades como individuos hay-. William James, en las Variedades de la experiencia religiosa (1902/1985), comenzó un pro- yecto que cuidadosamente soslayó "la religiosidad promedio" y apreció la diversidad entre individuos. Entre esta multitud de tipos de $\mathrm{Fe} o$ de maneras de ser religiosa/o, ¿cuáles se relacionan con las distintas formas de trabajar por la justicia?

\section{RELIGIÓN Y PREJUICIO}

En los años cuarenta y cincuenta, siguiendo el exordio de Freud (1927) contra la religión -y bajo la dominación de los conductistas-, la postura de muchos psicólogos hacia la religión consistió -en gran parte- en sospechas y cierto rechazo. Pensando que tal prejuicio podría matizar o manchar los lentes de los científicos sociales, era predecible que muchos psicólogos 
focalizaran su atención en la relación postulada entre religión y las actitudes prejuiciosas.

Los hallazgos de las primeras investigaciones acerca de la relación entre religiosidad y prejuicio, parecían confirmar las sospechas de los psicólogos: si se definiera la religiosidad operacionalmente como, la frecuencia (admitida) de asistencia a los servicios religiosos y se midiera el prejuicio por medio de la puntuación obtenida en escalas de actitudes hacia las minorías y grupos políticos minoritarios opositores, entonces, los que asistían obtendrían puntajes más altos que los que no asistían (Glock \& Stark, 1966).

Este resultado era consistente, por mucho que se usaran medidas de etnocentrismo, racismo, antisemitismo y así sucesivamente. Pero, en la gran mayoría de estas investigaciones, se trató la variable de la religiosidad como una variable dicotómica. Cuando se midió la frecuencia de asistencia por lo menos con tres niveles de participación -bajo, moderado y alto-, se obtuvo una información de mayor complejidad que antes: al fin y al cabo, la relación no era lineal, sino curvilínea, formando una curva en forma de una U-invertida (Gorsuch \& Aleshire, 1974; en Spilka et al., 2003, p. 458-459).

Allport y Ross (1977) se intrigaron por estos resultados y ponderaron la posibilidad de que esta función encerrara, por lo menos, dos motivos distintos de ser religiosa/o (o sea de asistir a servicios religiosos): una persona podría estar motivada intrínsecamente -en esto caso, se viviría la religión-; por otro lado, se podría estar motivado extrínsecamente, en tal caso, se utilizaría la religión. En el primer caso, la religión es en sí su propia meta; en el segundo, se usa la religión para alcanzar otras metas.

Parece que, las ásperas censuras de Freud se aplican, específicamente, a los que son religiosos en una manera extrínseca. De hecho, en más de una docena de investigaciones donde se comparó a las personas que fueran tipificadas como "religiosamente extrínsecas", con personas "religiosamente intrínsecas", en cada una, las extrínsecas consiguieron puntuaciones mas altas, en cualquiera de las medidas de prejuicio, que las intrínsecas (Batson, Flink, Schoenrade, et al., 1986). En estudios mas recientes, la religiosidad intrínseca se correlaciona también con varias medidas de salud mental, incluso con competencia y control y una conducta social apropiada; mientras la religiosidad extrínseca se correlaciona con ansiedad sobre la muerte y con inquietud y culpabilidad; y así sucesivamente (Batson, Schoenrade, \& Ventis, 1993, p. 269).

Se debe notar que, las preocupaciones serias y críticas sobre la validez de ambas escalas (también sobre la réplica del experimento original) han creado dudas sobre conclusiones tan sencillas. Por ejemplo, Allport y Ross propusieron una matriz en la cual hay personas de tipo intrínsecas, extrínsecas, no religiosas, y "religiosas de manera indistinta" (es decir, con puntuaciones altas en ambas escalas); sus sucesores han ignorado, por lo general, la posibilidad de un efecto interactivo entre "intrínseca" y "extrínseca." Hay otras críticas y dudas también (Spilka et al., 2003, 459-461); esta línea de debate se queda fuera del ámbito de esta discusión. Por lo menos, la moraleja hasta aquí parece ser que, algunos tipos de la $\mathrm{Fe}-$ i.e., los que involucran un compromiso total- están mas lejos de la injusticia, que otros -i.e., los que involucran utilizar la religión como una herramienta o medio-.

Armstrong (1993,) expresa este sentido en que un individuo puede utilizar la religión como un medio para sus propias metas:

"Un dios personal puede convertirse en una desventaja grave. Puede ser un mero ídolo, tallado en nuestra propia imagen, una proyección de nuestras necesidades, miedos, y deseos limitados. Podemos asumir que él ame lo que amamos y odie lo que odiamos; que apruebe nuestros prejuicios en vez de obligarnos a que los transcendamos. En lugar de inspirar la compasión que debería caracterizar todas las religiones avanzadas, este dios puede animar a que nosotros juzguemos, condenemos y excluyamos."

\section{RELIGIÓN Y ALTRUISMO}

Se debe dejar a un lado esta línea de investigación por el momento y considerar, en lugar de la intolerancia y prejuicio, la caridad o el altruismo. Se puede plantear la siguiente pregunta: ¿son "más simpáticas" las personas más religiosas que las menos religiosas?. Es decir, ¿está relacionada la religiosidad con las actitudes y conductas sociales?.

Un repaso de la literatura, revela un aumento gradual en la sofisticación con respecto a las definiciones operacionales utilizadas en investigaciones sucesivas. Entonces, se puede representar el crecimiento del entendimiento de 
esta relación como una función de cambios en la forma de medir las variables. Por un lado, se ha visto que la medida de la religiosidad ha cambiado, desde la frecuencia de la asistencia hacia unos estilos o maneras de ser religioso. Por otro lado, Batson y sus colegas (Batson \& Gray, 1981; Darley \& Batson, 1973) aumentaron el alcance de las definiciones operacionales del altruismo, desde medidas de autoinforme hacia medidas que incluían conductas observadas y objetivas. En un repaso de la literatura sobre investigaciones en ésta área, Batson, Schoenrade y Ventis (1993) describieron los resultados de cuatro grupos de investigaciones:

(1) Cuando se mide la religiosidad como frecuencia de asistencia y el altruismo por medio de instrumentos de autoinforme, se hallan (por lo general) correlaciones positivas aunque escasas (en nueve de nueve estudios).

(2) Cuando se mide la religiosidad como frecuencia de asistencia y el altruismo por medio de observaciones de conducta, no aparecen diferencias ni correlaciones significativas (solamente en uno de seis estudios se halló una diferencia significativa),

(3) Cuando se usan los estilos de religiosidad o sea las orientaciones religiosas de Allport y encuestas de autorreflexión, que muestran una inquietud acerca del bienestar de los demás o un cálculo de la extensión de la conducta de ayuda de uno mismo, de nuevo, consistentemente, hay una relación positiva entre la religiosidad intrínseca y cualquier medida de altruismo. Por otro lado, hay una correlación negativa o inversa entre el altruismo y la religiosidad extrínseca.

(4) En cuanto a la vinculación entre orientaciones religiosas y conductas prosociales, los resultados no son consistentes; también hasta ahora, las investigaciones no son muchas. Una serie de investigaciones de Bolt (1982), revelaron una correlación significativa -aunque bajaen la cual los intrínsecos obtuvieron puntajes más altos en medidas conductuales del altruismo. Pero Batson rechazó este hallazgo, argumentando que el costo de ayudar en estos estudios fue menos que el mínimo. Entonces, los motivos en la conducta de ayuda -así como el propio altruismo en los casos uno y tres- parecieron involucrar el deseo de manifestarse como agradable y simpático.

En una investigación clásica, Darley y Batson (1973) realizaron un experimento para determinar la naturaleza de la relación entre religiosi- dad y conducta de ayuda (en vez de las respuestas de autoinforme). Convocaron a 40 seminaristas (todos varones), asumiendo que todos fueran "por lo menos moderadamente religiosos." Cada seminarista cumplimentó las escalas de religiosidad intrínseca y extrínseca, también un tercer instrumento desarrollado por Batson, titulado la "Búsqueda" (Quest). Esta escala tiene que ver con una tendencia a enfrentar las preguntas existenciales en toda su complejidad, mientras es poco permeable a soluciones o contestaciones fáciles y oportunas.

Después de cumplimentar las escalas, los seminaristas fueron enviados uno por uno (cada 20 minutos), a través del recinto, para dar una conferencia. Los investigadores incluyeron dos variables experimentales: (a) les dijeron a una parte $(50 \%)$ que la conferencia tenía que ver con la parábola del Buen Samaritano; a los demás se les dijo que tenía que ver con oportunidades profesionales. (b) a una mitad les dijeron que la conferencia comenzaba pronto; a la otra, no. Cada seminarista, al cruzar el recinto encontró a una "víctima" (un socio de los investigadores) que parecía estar en apuros. La variable dependiente era, simplemente, si el seminarista paraba y ayudaba a la víctima o no.

Solamente 16 de los 40 pararon para ayudar. No sorprende que ninguna de los tres variables de la disposición - ni la motivación intrínseca, ni la extrínseca, ni la búsqueda- produjeran resultados diferentes. Solamente la situación de apuro influyó en la probabilidad de ayudar o no. La sorpresa -no anticipada por los investigadores- tuvo que ver (en vez de si se ayudara o no) con el estilo de ayudar por parte de los 16 que pararon. El socio relató a los investigadores, inicialmente, que en el experimento algunos de quienes pararon no aceptaron su rechazo por la ayuda: es decir, insistieron en ayudarle, no importando lo que les dijera. Sabiendo que otro seminarista caminaría por el sendero cada 20 minutos, esto creó un problema logístico para el comprometido socio!

Darley y Batson (1973) volvieron a analizar los datos utilizando solo los dieciséis buenos samaritanos; se hicieron cargo de las escalas de orientación religiosa que predecían el estilo de ayuda. Los individuos con altos niveles de orientación intrínseca se inclinaron a ser "auxiliadores persistentes," que insistieron en ayudar, sucediera lo que sucediera. Por otro lado, los que tenían puntuaciones mas altas 
en la escala de búsqueda, se inclinaron a ser "auxiliadores tentativos," es decir, ofrecieron su ayuda pero a la vez respondieron con sensibilidad, según los deseos y necesidades comunicados por la "víctima." Especularon que, la diferencia en el estilo de ayudar y responder, proviene de una diferencia en la motivación para ayudar. ¿Por qué ayudaron estos buenos samaritanos? Argumentaron que un motivo posible tiene que ver con la necesidad de "parecer bueno": el auxilio persistente ayuda, porque el acto de ayudar confirma -o valida- su deseo de ser una buena persona, a los ojos de sus pares o a los ojos de un "observador ideal," su dios o un estándar moral. En tales casos, puede ser que la víctima sencillamente ofrece una ocasión u oportunidad para llevar a cabo una obligación. Los investigadores notaron que la escala de religiosidad intrínseca, es influida por la forma de responder de manera "socialmente correcta"; es decir, muchos críticos han argumentado que las puntaciones en esta escala reflejan, no una tendencia a vivir su religión o sea una madurez en su religiosidad, sino más un deseo de parecer religioso. Es decir, la escala tiene "respuestas correctas." Entonces, no es sorprendente que los "religiosos intrínsecos" tienden a ser los mismos que los "auxiliadores persistentes."

Algunas críticas de esta línea de investigación han propuesto que, aunque sus medidas de conducta de ayuda/auxilio son superiores a las medidas de introspección, son limitadas, en vista de que se enfocan exclusivamente en tal conducta en situaciones urgentes que exigen una respuesta espontánea; es decir, las medidas tienen que ver con la manera de responder "en el momento", cuando se enfrenta con alguien en apuros (Bernt, 1989). ¿Qué pasa cuando se enfoca en respuestas "no-urgentes" y "no-espontáneas o planeadas"; por ejemplo, si alguien decide pasar un año después de graduarse como voluntario en el Cuerpo de Paz o dedicar tres horas cada semana a trabajar con los desamparados?. Bernt (1989), obtuvo resultados que apoyaron tal hipótesis: los factores de disposición realmente predicen tales conductas, a diferencia de los resultados de Batson. A la vez, de nuevo, que la escala de Búsqueda parece predecir este tipo de conducta mejor que las otras escalas. La pregunta que surge entonces es: ¿qué motiva al individuo con una orientación de Búsqueda a buscar oportunidades para ayudar a los demás?

\section{RELIGIÓN Y PREJUICIO. MÁS ALLÁ}

Regresando al primer tema, se puede complicar aun más la vinculación entre religión y prejuicio, por medio de (a) aumentar la lista de medidas de religión y (b) considerar varios objetos de prejuicio.

Se mencionó antes que, las críticas a las escalas de Allport y Ross han hecho -en algunos campos de la psicología- desestimar el uso de las mismas, sugiriendo que han durado más que su utilidad (Pargament, 1992). Como respuesta a estas críticas, ha ocurrido un aumento del uso de otras escalas para investigar el tema. Hay dos que se destacan: la escala de Búsqueda de Batson (mencionada antes) y una variedad de escalas de fundamentalismo religioso o autoritarismo derechista. El concepto de fundamentalismo se introdujo con William James, bien temprano en el siglo pasado; lo describió como un estilo rígido y dogmático que podría ser asociado con intolerancia y prejuicio (W. James, 1902/1997, p. 266).

Investigaciones en el período que va de los años cincuenta al ochenta, parecieron establecer una correlación fuerte entre el fundamentalismo religioso y el prejuicio, utilizando una variedad de definiciones operacionales. En los años noventa, Altemeyer y Hunsberger (1992), reformularon el concepto en su Test "Right-Wing Authoritarianism" (Autoritarismo Derechista), con las presunciones consiguientes: (a) hay una sola suma de enseñanzas religiosas que contiene la verdad esencial acerca de la humanidad y la deidad; (b) esta verdad es opuesta a las fuerzas de la maldad, contra la cual se debe luchar; (c) se debe seguir esta verdad según las prácticas tradicionales del pasado y (d) quienes creen y obedecen estas enseñanzas fundamentales tienen una relación especial con la deidad.

Hunsberger y Jackson (2005), desarrollaron un meta-análisis de dieciséis investigaciones que usaron las cuatro escalas y consideraron los objetos de prejuicio siguientes: (a) Grupos Raciales/étnicos; (b) Homosexuales / Lesbianas; (c) Mujeres; (d) Comunistas y (e) Grupos Religiosos Marginalizados. Por lo general, puntajes en la escala Quest / Búsqueda se correlacionaron negativamente con todos los tipos de intolerancia; los de la escala Autoritarismo se correlacionaron positivamente con todos los tipos (excepto contra grupos raciales/ étnicos, en la cuál de 11 estudios, 6 revelaron 
una relación positiva y 5 no revelaron relación alguna).

En cuanto a la escala Extrínseca, los resultados estaban mezclados, pero había una tendencia leve de asociaciones positivas. La escala Intrínseca da resultados que apoyan las sospechas de Batson et al. (1986): hay una correlación negativa (inversa) entre esta escala y medidas de prejuicio racial (prejuicio condenado por la mayoría de religiones); mientras hay una correlación positiva entre la escala y medidas de prejuicio contra minorías sexuales (nocondenado).

Se puede imaginar que la dinámica de responder de manera socialmente correcta, mencionada antes, juega un papel significativo cuando se considera que, en la época después de los años sesenta, muchos tipos de prejuicios en particular el racismo- han sido suprimidos o se convirtieron en encubiertos. El resultado de este cambio social y el deseo personal de mantener una autoimagen no-prejuiciosa y humanitaria, puede hacer que mucha gente tienda a menospreciar sus propios niveles de prejuicio. Entonces, hay una variedad de teorías de dos factores; ejemplos que incluyen el racismo aversivo (Gaertner \& Dovidio, 1986); racismo ambivalente (Katz \& Hass, 1988) y racismo simbólico (Sears, 1988).

Para medir la discriminación de manera verdadera y válida, los psicólogos han desarrollado nuevos tests que tratan de controlar la dinámica de "parecer socialmente correcto." Por ejemplo, Batson distinguió entre "racismo abierto" y "racismo escondido o encubierto" utilizando medidas conductuales que se podían observar. Como se puede predecir, los puntajes en la escala de Búsqueda correlacionaban negativamente con cualquiera de ambos (abierto o escondido); en la escala Extrínseca, la correlación fue positiva (pero no significativa) y en la Intrínseca, negativa con la medida de Racismo abierto, mientras positiva con la medida de Racismo Escondido o Encubierto.

Un conjunto de investigaciones, que usaron varias muestras en Europa y en el Caribe, también con grupos religiosos de judíos, hindúes y musulmanes, obtuvieron resultados consistentes con los hallazgos de Altemeyer y Hunsberger. Quizás lo más interesante es que, Snook y Gorsuch (1985), usando muestras de blancos en Sudáfrica descubrieron que, entre respondientes afiliados a iglesias Africanas (que apoyaban el Apartheid), la Religiosidad Intrín- seca tenía una relación positiva o directa con el racismo; entre los pertenecientes a iglesias inglesas (que no apoyaban el Apartheid), dicha relación era negativa.

\section{RELIGIÓN, ALTRUISMO Y JUSTICIA}

Hay que regresar una última vez, a la relación entre religión y altruismo. Un estudio realizado por Jackson y Esses (1997), indagó el papel de cada uno de los cuatro aspectos de la orientación religiosa -mencionado antes- al decidir si se debe ayudar o no el otro, como función de su mérito. Se puede considerar (al igual que en apartados anteriores), sobre quien es el objeto de la ayuda. Según la teoría de la atribución, si pienso que él/ella es culpable por su propio problema, yo sentiría menos obligación de ayudar o rescatarle. En tal situación, respondería con una reprimenda; una sugerencia sobre que él/ella tiene que hacerse responsable de sus propias acciones.

En dicho estudio, realizado con alumnos universitarios canadienses, la tarea involucró leer pasajes sobre el problema de desempleo en Canadá. Después, los alumnos respondieron encuestas que midieron atribuciones y maneras de ayudar. Pudieron atribuir el desempleo a factores internos de los desempleados (por ejemplo, pereza) o atribuirlo a factores externos (por ejemplo, el sistema económico). Las respuestas de ayuda se clasificaron según cuatro modelos:

- Modelo moral - que involucra censura, crítica y el juzgar;

- Modelo de instrucción - que involucra alentar a la ayuda de las autoridades;

- Modelo remedial - que involucra el ofrecimiento de ayuda directa y

- Modelo compensatorio - que involucra el empoderamiento para ayudarse a sí mismo

Se puede ver que, los primeros dos vienen de una tendencia a "culpar a la víctima"; los últimos dos, de una tendencia a culpar a las circunstancias y sistemas, externos a la víctima.

La variable manipulada en este estudio fue el nivel de amenaza a los valores del sujeto, que representa el desempleado. En la condición de amenaza, los participantes leyeron pasajes que incluían a homosexuales o madres no casadas. La condición de no-amenaza incluyó indios canadienses y alumnos universitarios. Los resultados revelaron que, los participantes con puntajes altos en la escala de 
fundamentalismo tendieron a "culpar a la víctima” (y entonces censurar o juzgar, en vez de ayudar) en las condiciones que amenazaron sus valores, pero no en situaciones no amenazantes. No había diferencia entre las respuestas de los alumnos con puntajes bajos en la escala de fundamentalismo.

$Y$ que pasó en cuanto a los alumnos con orientación de Búsqueda? O de orientación Intrínseca? Desafortunadamente, Jackson y Esses no incluyeron estas escalas en su estudio. Sin embargo, en dos estudios bien parecidos, realizados por Batson et al. (1999, 2001) con alumnos universitarios, se hallaron las mismas diferencias mencionadas anteriormente para los "Intrínsecos Altos" (es decir, la tendencia de "culpar al víctima" o de no ayudarlo) cuando la víctima representaba una amenaza.

\section{CONCLUSIONES}

Como resumen, podemos subrayar algunas implicancias del tema, a modo tentativo:

\section{Asuntos referidos a la Metodología.}

Hay varias propuestas de mejoramiento en esta línea de investigación. Todavía hay un uso excesivo de muestras de alumnos. El trabajo de Batson y sus colegas ha proporcionado mucho, pero ahora se necesita replicar esos estudios utilizando varios grupos. Hay que seguir el ejemplo de Batson, en complementar el uso de encuestas con observaciones de conducta, tanto, en cuanto al altruismo como al prejuicio. Además de categorizar respuestas altruistas en términos de sus objetos, sería ventajoso desarrollar una taxonomía de tales respuestas. La definición operacional de "conducta de auxilio/ ayuda", ha incluido: devolver cartas perdidas; donación de sangre; dar dinero a extranjeros; ofrecerse como voluntario para leer a ciegos y así sucesivamente; y todas estas definiciones se han tratado como homogéneas o iguales. Se precisa una "taxonomía" que distinga -por lo menos- el corto plazo del largo plazo; lo costoso de lo poco costoso; lo espontáneo de lo planificado; lo reactivo de lo proactivo.

\section{Compromiso Religioso.}

Los estudios de Allport, también de sus sucesores, han establecido la importancia de tener un compromiso profundo y sostenido con una Fe personal, que atraviesa toda la vida. A pesar de que la escala de motivación intrínseca tiene sus debilidades, es claro que el compro- miso religioso es una condición necesaria, aunque no una condición suficiente, de la lucha por la justicia. Lo que se añade con la escala de búsqueda es el aspecto dinámico de una Fe que forje la justicia. Si alguien está comprometido con una Fe o sea una perspectiva religiosa que no vive, que no admite la posibilidad de cambiar, el compromiso puede hacerse, no en una $\mathrm{Fe}$ madura, sino en una Fe impedida. O sea, aunque parece una blasfemia a primera vista, uno puede hacerse adicto a su religión si no incluye el aspecto de búsqueda y esta adicción produce inconsistencias, aún obstáculos, en la lucha por la justicia (Arterburn \& Fèlton, 2001, p. 7).

\section{Estilos y Motivos para Ayudar.}

Hay un cuento de un padre que trabaja, hombro con hombro- con sus hijos, en un albergue para los desamparados. El padre tiene mucha satisfacción por su trabajo y con la experiencia que él proporciona a sus hijos. En un momento emotivo, anuncia efusivamente "ojalá que mis nietos y mis bisnietos puedan continuar este trabajo por mucho tiempo, después de mi muerte." Esto es ayudar persistentemente, no tentativamente. Refleja la caridad sin un sentido de la justicia. Muchas veces es el motivo primario para comenzar una carrera de servicio. No debe ser el único motivo para continuar tal carrera. Las experiencias "cara a cara" con los desventurados deben fomentar una sensibilidad, mas allá de "noblesse oblige," una sensibilidad de solidaridad. En este sentido, la lucha por la justicia requiere una Fe. En este contexto, debe ser una vinculación íntima entre dimensiones verticales y horizontales del amor cristiano, en la cual la caridad produce en nosotros un deseo de identificarnos con los pobres, de ayudarlos y entonces, buscar la justicia por medio de transformaciones tanto personales como sociales (Aron \& McLaughlin-Volpe, 2002; Arrupe, 1980). Investigaciones futuras deberian incluir en su diseño tales instrumentos, como "La Creencia en un Mundo Justo" (Lipkus, 1991) para explorar mas allá dicha vinculación.

El papel del "Otro".

Investigaciones más recientes que han utilizado escalas de fundamentalismo y de autoritarismo, han mostrado tanto los riesgos de las "religiones tribales" como los beneficios de las prescripciones humanistas de iglesias específicas y de tradiciones religiosas más amplias. Es obvio que, la vinculación (en sentido psicológico, tanto como en un sentido mas amplio) entre 
Fe y justicia, tiene que ver con las personas religiosas (como los enfatizados por la mayoría de las investigaciones psicológicas) y también tiene que ver con los contextos sociales de sus actividades religiosas. Las iglesias de hoy proveen oportunidades para "ensayar el evangelio" con los demás, en una multitud de modos. Debemos estudiar, en el futuro, nuestras imágenes de "los demás," también de nuestras relaciones con ellos y considerar como eso se vincula a nuestras propias imágenes de Dios y de nuestra relación con El (Lawrence, 1997).

Escasez de Medidas de Compromiso con la Justicia (en vez del Altruismo).

Claramente los psicólogos se han hecho más creativos y astutos en sus evaluaciones; no obstante, podría ser que no han tocado ni se han acercado, a la esencia del tema de la lucha por la justicia. Las medidas conductuales de Batson y otros son importantes, puesto que se extienden más allá de los problemas de las medidas de los auto-informes. Sin embargo, casi todos involucran la ayuda en situaciones de emergencia, ayuda de corto plazo o ayuda como respuesta a condiciones creadas en contextos artificiales. Hay muy pocas investigaciones que consideren, por ejemplo, correlaciones entre orientación religiosa y activismo político (Green, 2003) o actitudes sobre políticas públicas (por ejemplo, acerca de la inmigración; Bernt, 2005).

El mártir Martin Luther King dijo que "el arco del universo moral es largo, pero dobla hacia la justicia." Si él tiene razón, precisamos encontrar maneras de pensar sobre actitudes y conductas, que representan un compromiso con la justicia (y también instrumentos psicológicos para medirlas).

\section{REFERENCIAS}

Allport, G. W. (1954). The nature of prejudice. Cambridge, MA: Addison-Wesley.

Allport, G. W., \& Ross, J. M. (1967). Personal religious orientation and prejudice. Journal of Personality and Social Psychology, 5, 432-443.

Altemeyer, B. \& Hunsberger, B. (1992). Authoritarianism, religious fundamentalism, quest, and prejudice. International Journal for the Psychology of Religion, 2, 113-133.

Armstrong, K. (1993). A history of God: The 4,000year quest of Judaism, Christianity and Islam. New York: Alfred A Knopf, Inc.

Aaron, A. \& McLaughlin-Volpe, T. (2002). Including others in the self. In C. Sedikides \& M. B. Brewer
(Eds.), Individual self, relational self, collective self (pp. 89-108). Philadelphia: Psychology Press.

Arrupe, P. (1980). Justice with faith today. Ed. J. Aixala. St. Louis, MO: Institute of Jesuit Sources.

Arterburn, S., \& Fèlton, J. (2001). Toxic faith: Experiencing healing over painful spiritual abuse. New York: Random House.

Batson, C. D., Eidelman, S. H., Higley, S. L., \& Russell, S. A. (2001). "And who is my neighbor?": II. Quest religion as a source of universal compassion. Journal for the Scientific Study of Religion, 40, 39-50.

Batson, C. D., Flink, C. H., Schoenrade, P. A., Fultz, J., \& Pych, V. (1986). Religious orientation and overt versus covert racial prejudice. Journal of Personality and Social Psychology, 50 (1), 175181.

Batson, C. D., Floyd, R. B., Meyer, J. M., \& Winner, A. L. (1999). "And who is my neighbor?:" Intrinsic religion as a source of universal compassion. Journal for the Scientific Study of Religion, 38 (4), 445-457.

Batson, C. D., \& Gray, R. A. (1981). Religious orientation and helping behavior: Responding to one's own or to the victim's needs? Journal of Personality and Social Psychology, 40, 511-520.

Batson, C. D., Schoenrade, P., \& Ventis, W. L. (1993). Religion and the individual: A socialpsychological perspective. New York: Oxford University Press.

Batson, C. D., \& Ventis, W. L. (1982). The religious experience: A social-psychological perspective. New York: Oxford University Press.

Bernt, F. M. (1989). Being religious and being altruistic: A study of college service volunteers. Personality and Individual Differences, 10, 663669.

Bernt, F. M. (1999). Religious commitment, attributional style, and gender as predictors of undergraduate volunteer behavior. Journal of Psychology and Theology, 27, 3, 261-272.

Bernt, F. M. (2005). Abrazar al Forastero entre Nosotros: Actitudes Estadounidenses Sobre la Inmigración Latinoamericana. Conferencia presentada en el Congreso de la Sociedad Interamericana de Psicólogos, Buenos Aires, Argentina.

Darley, J. M., \& Batson, C. D. (1973). "From Jerusalem to Jericho": A study of situational and dispositional variables in helping behavior. Journal of Personality and Social Psychology, 27, 100-108.

Duck, R. J., \& Hunsberger, B. (1999). Religious orientation and prejudice: The role of religious 
proscription, right-wing authoritarianism, and social desirability. International Journal for the Psychology of Religion, 9 (3), 157-189.

Freud, S. (1961). The future of an illusion (J. Strachey, Trans.). New York: Norton. (original work published 1927).

Gaertner, S. L., \& Dovidio, J. F., (1986). The aversive form of racism. In J. F. Dovidio \& S. L. Gaertner (Eds.), Prejudice, discrimination, and racism (pp. 61-89). Orlando, FL: Academic Press.

Gorsuch, R. L., \& Aleshire, D. (1974). Christian faith and ethnic prejudice: A review and interpretation of research. Journal for the Scientific Study of Religion, 13, 281-307.

Green, J. C. (2003). A liberal dynamo: The political activism of the Unitarian-Universalistic clergy. Journal for the Scientific Study of Religion, 42 (4), 577-590.

Hunsberger, B., \& Jackson, L. (2005). Religion, meaning, and prejudice. Journal of Social Issues. 61 (4), 807-826.

Jackson, L. M., \& Esses, V. M. (1997). Of scripture and ascription: The relation between religious fundamentalism and intergroup helping. Personality and Social Psychology Bulletin, 23, 893-906.

James, W. (1985). The varieties of religious experience. Cambridge, MA: Harvard University Press. (Original work published 1902).
Lawrence, R. T. (1997). Measuring the image of God: The God Image Inventory and the God Image Scales. Journal of Psychology and theology, 25 (2), 214-226.

Lipkus, I. (1991). The construction and preliminary validation of a Global Belief in a Just World Scale and the exploratory analysis of the Multidimensional Belief in a Just World Scale. Personality and Individual Differences, 12, 1171-1178.

Pargament, K. (1992). Of means and ends: Religion and the search for significance. International Journal for the Psychology of Religion, 2, 201-229.

Pettigrew, T. F. (1998). Generalized intergroup contact effects on prejudice. Personality and Social Psychology Bulletin, 23, 173-185.

Sears, D. O., \& Henry, P. J. (2003). The origins of symbolic racism. Journal of Personality and Social Psychology, 85 (2), 259-275.

Sears, D. O. (1988). Symbolic racism. In P. Katz \& D. Taylor (Eds.), Eliminating racism: Profiles in controversy (pp. 53-84). New York: Plenum Press.

Snook, S. C., \& Gorsuch, R. L. (1985). Religion and racial prejudice in South Africa. Paper presented at the annual convention of the American Psychological Association, Los Angeles.

Spilka, B., Hood, R. W., Jr., Hunsberger, B., \& Gorsuch, R. (2003). The psychology of religion: An empirical approach. Third Editions. New York: The Guilford Press. 